Article

\title{
Type-1 Fuzzy Sets and Intuitionistic Fuzzy Sets ${ }^{\dagger}$
}

\author{
Krassimir T. Atanassov 1,2 \\ 1 Department of Bioinformatics and Mathematical Modelling, Institute of Biophysics and Biomedical \\ Engineering, Bulgarian Academy of Sciences, Acad. G. Bonchev Str., Bl. 105, Sofia 1113, \\ Bulgaria; krat@bas.bg \\ 2 Intelligent Systems Laboratory, Prof. Asen Zlatarov University, Bourgas 8010, Bulgaria \\ † In memory of Prof. Lotfi Zadeh (1921-2017).
}

Received: 3 July 2017; Accepted: 31 August 2017; Published: 13 September 2017

\begin{abstract}
A comparison between type-1 fuzzy sets (T1FSs) and intuitionistic fuzzy sets (IFSs) is made. The operators defined over IFSs that do not have analogues in T1FSs are shown, and such analogues are introduced whenever possible.
\end{abstract}

Keywords: intuitionistic fuzzy set; intuitionistic fuzzy operator; Type 1 Fuzzy Sets

\section{Introduction}

In 1965, Lotfi Zadeh introduced the concept of fuzzy sets [1]. They are now one of the most serious and prospective directions for the development of Georg Cantor's set theory. In spite of the concerns and critical remarks against fuzzy sets voiced by some of the most prominent specialists in the area of mathematical logic in the second half of the 1960s, fuzzy sets have been firmly established as a fruitful area of research, as well as of a tool for the evaluation of different objects and processes in nature and society. Soon after their launch, fuzzy sets became an object of extensions by themselves. Chronologically, the first of these extensions, L-fuzzy sets, were introduced by Goguen in 1969 [2]. The second extension was from Zadeh [3], who introduced the idea of interval-valued fuzzy sets. The third extension is the rough sets, which were defined by Z. Pawlak in 1981 [4,5]. The fourth was intuitionistic fuzzy sets (IFSs), introduced in 1983 [6]. In recent years, many other extensions of fuzzy sets have been proposed. It is now clear that a significant part of these are trivial modifications of other existing fuzzy set extensions which have been "re-branded" under new names.

In recent years, some authors have introduced the term "type-1 fuzzy set" (T1FS) as a synonym of Zadeh's fuzzy set (e.g., [7-9]), emphasizing that T1FSs are the basis of the fuzzy sets extensions. Preserving this name, in the present paper, we discuss what is common and what is different between T1FSs and IFSs.

In Section 2 , we discuss the basic concepts in IFS theory that do not have analogues in T1FS theory and the reasons for this. In Section 3, we discuss the possibility for the transformation of some concepts from IFSs theory to T1FSs theory. So, we show some new directions for development of T1FS theory. The author is not aware of similar research.

The author would like to mention that his first research in the area of IFS was based on A. Kaufmann's book [10], whose Russian translation was the first book on fuzzy sets that appeared in Bulgaria in the early 1980s. For this reason, the theory of IFSs uses the notation of [10].

\section{What Is There in IFS Theory That Has No Analogue in T1FS Theory?}

Below, following [11,12], we give the definitions of the basic concepts and the basic operations, relations, and operators over IFSs, and discuss which of them do and which do not have analogues in T1FS theory. 
Let us have a fixed universe $E$ and its subset $A$. The set

$$
A^{*}=\left\{\left\langle x, \mu_{A}(x), v_{A}(x)\right\rangle \mid x \in E\right\}
$$

where

$$
0 \leq \mu_{A}(x)+v_{A}(x) \leq 1
$$

is called IFS and functions $\mu_{A}: E \rightarrow[0,1]$ and $v_{A}: E \rightarrow[0,1]$ represent the degree of membership (validity, etc.) and non-membership (non-validity, etc.), respectively. In IFSs, we can also define the function $\pi_{A}: E \rightarrow[0,1]$ by

$$
\pi(x)=1-\mu(x)-v(x),
$$

which corresponds to the degree of indeterminacy (uncertainty, etc.).

For brevity, we shall write below $A$ instead of $A^{*}$, whenever possible.

Obviously, for every T1FS $A: \pi_{A}(x)=0$ for each $x \in E$, and this set has the form $\left\{\left\langle x, \mu_{A}(x), 1-\mu_{A}(x)\right\rangle \mid x \in E\right\}$.

By analogy with the T1FSs, IFSs have the following geometrical interpretation (see Figures 1 and 2).

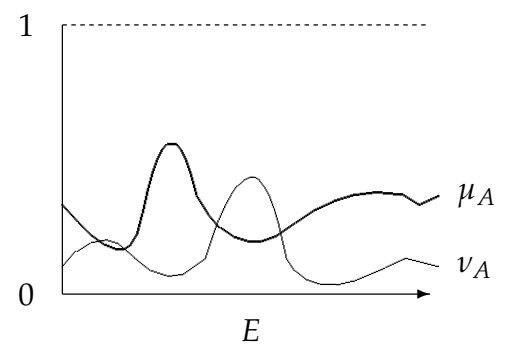

Figure 1. First geometrical interpretation-first form.

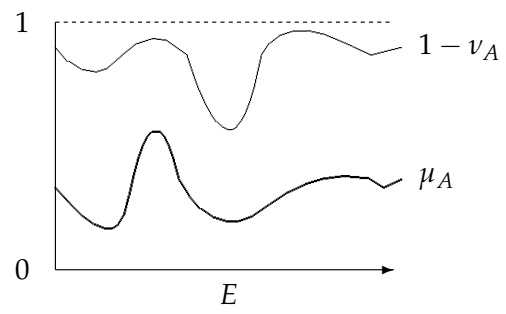

Figure 2. First geometrical interpretation—second form.

In the T1FS-case, in both figures, the line for $v$ is missing and they coincide.

The IFSs have another geometrical interpretation (see Figure 3) that analogously can be transformed to the T1FS-case with omission of the line for $v$. It is the first geometrical interpretation in circle.

The most practically useful geometrical interpretation of the IFSs (see Figure 4) makes no sense for T1FS, since the elements of such a set are only points projected onto the hypotenuse of the intuitionistic fuzzy interpretation triangle.

The situation with the next geometrical interpretation of the IFSs is similar (see Figures 5-8). The interpretation in Figure 7 was introduced by Danchev in [13], while Szmidt and Kacprzyk constructed the three-dimensional geometrical interpretation in Figure 8 in [14-16].

In [17], the latest geometrical interpretation is introduced (see Figure 9), where $p \in E$. In it, the horizontal section and the two vertical sections have length of 1 . The section determines the two boundary points that have ordinates with lengths $v(p)$ and $\mu(p)$. 
In this new interpretation, the central point-marked in Figure 10 by $\bullet$ with coordinates $\left\langle\frac{1}{2}, \frac{1}{2}\right\rangle$-plays an important role. When a section passes through it, then this section corresponds to a T1FS-element (i.e., one, for which $\pi(p)=0$ ).

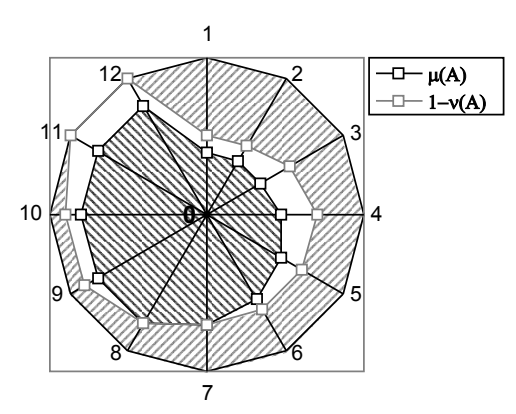

Figure 3. V. Atanassova's geometrical interpretation.

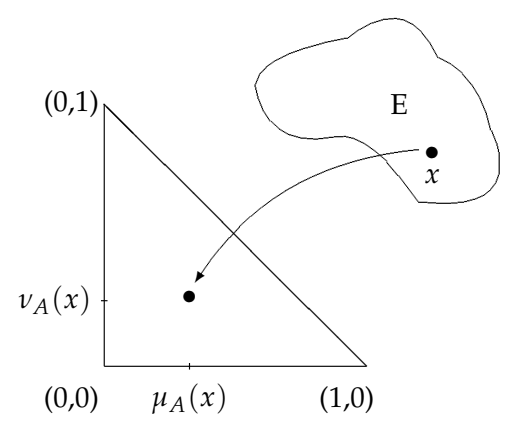

Figure 4. Second geometrical interpretation.

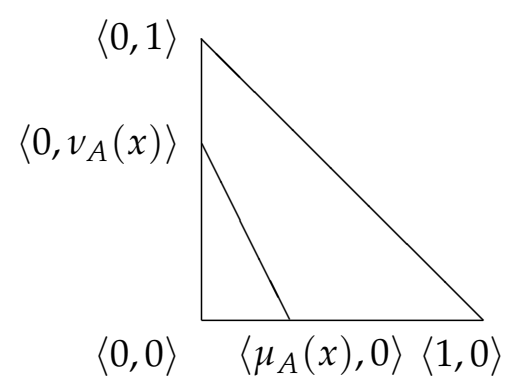

Figure 5. Third geometrical interpretation.

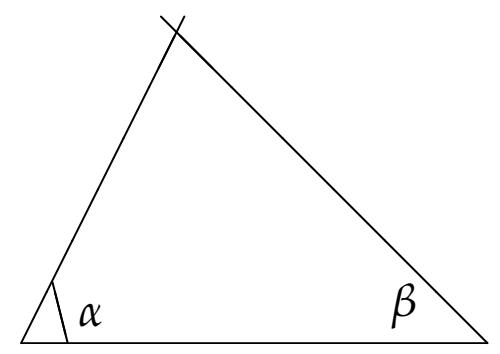

Figure 6. Fourth geometrical interpretation, where $\alpha=\pi \mu_{A}(x), \beta=\pi v_{A}(x)$ and here $\pi=3.14 \ldots$ 


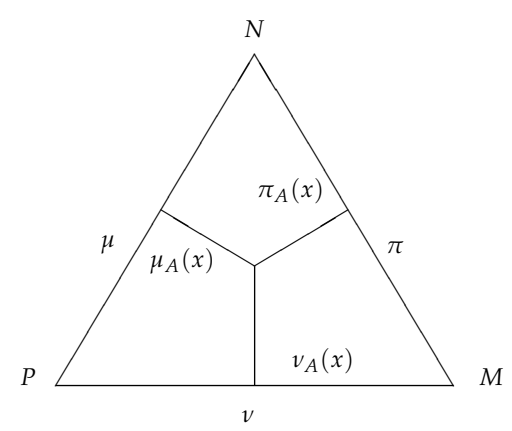

Figure 7. S. Danchev's geometrical interpretation.

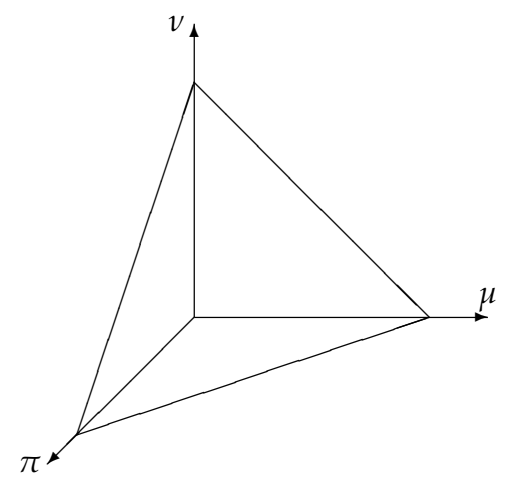

Figure 8. Szmidt and Kacprzyk's geometrical interpretation.

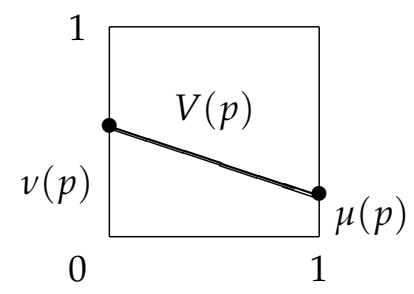

Figure 9. Fifth geometrical interpretation.

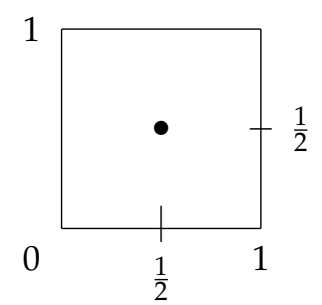

Figure 10. Central point in the fifth geometrical interpretation.

It is clear that the basic IFS-operations and relations can be transformed (reduced) to T1FS-operations. Such operations are, e.g.,

$$
\begin{aligned}
A \subset B \quad \text { iff } \quad(\forall x \in E) & \left(\left(\mu_{A}(x) \leq \mu_{B}(x) \& v_{A}(x)>v_{B}(x)\right)\right. \\
& \vee\left(\mu_{A}(x)<\mu_{B}(x) \& v_{A}(x) \geq v_{B}(x)\right) \\
& \left.\vee\left(\mu_{A}(x)<\mu_{B}(x) \& v_{A}(x)>v_{B}(x)\right)\right) ;
\end{aligned}
$$




$$
\begin{aligned}
A \subseteq B \quad \text { iff } \quad & (\forall x \in E)\left(\mu_{A}(x) \leq \mu_{B}(x) \& v_{A}(x) \geq v_{B}(x)\right) ; \\
A=B \quad \text { iff } \quad & (\forall x \in E)\left(\mu_{A}(x)=\mu_{B}(x) \& v_{A}(x)=v_{B}(x)\right) ; \\
\neg A= & \left\{\left\langle x, v_{A}(x), \mu_{A}(x)\right\rangle \mid x \in E\right\} ; \\
A \cap B= & \left\{\left\langle x, \min \left(\mu_{A}(x), \mu_{B}(x)\right), \max \left(v_{A}(x), v_{B}(x)\right)\right\rangle \mid x \in E\right\} ; \\
A \cup B= & \left\{\left\langle x, \max \left(\mu_{A}(x), \mu_{B}(x)\right), \min \left(v_{A}(x), v_{B}(x)\right)\right\rangle \mid x \in E\right\} ; \\
A+B= & \left\{\left\langle x, \mu_{A}(x)+\mu_{B}(x)-\mu_{A}(x) \times \mu_{B}(x), v_{A}(x) \times v_{B}(x)\right\rangle \mid x \in E\right\} ; \\
A \times B= & \left\{\left\langle x, \mu_{A}(x) \times \mu_{B}(x), v_{A}(x)+v_{B}(x)-v_{A}(x) \times v_{B}(x)\right\rangle \mid x \in E\right\} ; \\
A @ B= & \left\{\left\langle x, \frac{\mu_{A}(x)+\mu_{B}(x)}{2}, \frac{v_{A}(x)+v_{B}(x)}{2}\right\rangle \mid x \in E\right\} ; \\
A \rightarrow B= & \left\{\left\langle x, \max \left(v_{A}(x), \mu_{B}(x)\right), \min \left(\mu_{A}(x), v_{B}(x)\right)\right\rangle \mid x \in E\right\},
\end{aligned}
$$

defined for every two IFSs $A$ and $B$ (see [11,12]). The author was is only not aware of an operation over T1FS analogous of operation @ with the form

$$
A @ B=\left\{\left\langle x, \frac{\mu_{A}(x)+\mu_{B}(x)}{2}\right\rangle \mid x \in E\right\},
$$

where $A$ and $B$ are T1FSs.

Now, in [12,18], 53 different operations of intuitionistic fuzzy negation and 185 different operations of intuitionistic fuzzy implications are described. New 4 implications are given in [19-22]. The above implication is one of them. It has a T1FS-analogue, while approximately all the rest do not, but an analogy can be made with the above-mentioned operation @. The situation with the intuitionistic fuzzy negations is similar.

In IFS theory, there are some other operations that we do not discuss here because (at least at the moment) they do not have any practical application.

Now, there are four groups of operators, defined over IFSs. Below, we will shortly describe them and will discuss the possibility for them to obtain T1FS-forms.

As I wrote in [11,12], my research in IFSs started in February 1983 as a mathematical game, in which I transformed T1FS-operations to the above-described form of $A^{*}$. However, only when I found analogues of the two standard modal operators for the new type of sets did I understand that these sets are essentially different from the already existing T1FSs. George Gargov (1947-1996) invited me to call the new object an "intuitionistic fuzzy set", because of its correspondence to Brouwer's idea for intuitionism (see $[23,24])$. The first two modal operators over IFS $A$ have the form

$$
\begin{aligned}
& \square A=\left\{\left\langle x, \mu_{A}(x), 1-\mu_{A}(x)\right\rangle \mid x \in E\right\}, \\
& \diamond A=\left\{\left\langle x, 1-v_{A}(x), v_{A}(x)\right\rangle \mid x \in E\right\} .
\end{aligned}
$$

When the second geometrical interpretation (Figure 4) was constructed, it became clear why these two operators lose their sense if we try to define them over T1FS. This is so, because for each IFS $A$ :

$$
\square A \subset A \subset \diamond A,
$$

while, if $A$ is a T1FS

$$
\square A=A=\diamond A .
$$

Over the years, the two operators $\square$ and $\diamond$ have been the object of a series of extensions, some of which are mentioned below (see [12]): 


$$
\begin{aligned}
& D_{\alpha}(A)=\left\{\left\langle x, \mu_{A}(x)+\alpha \times \pi_{A}(x), v_{A}(x)+(1-\alpha) \times \pi_{A}(x)\right\rangle \mid x \in E\right\}, \\
& F_{\alpha, \beta}(A)=\left\{\left\langle x, \mu_{A}(x)+\alpha \times \pi_{A}(x), v_{A}(x)+\beta \times \pi_{A}(x)\right\rangle \mid x \in E\right\}, \\
& \text { where } \alpha+\beta \leq 1, \\
& G_{\alpha, \beta}(A)=\left\{\left\langle x, \alpha \times \mu_{A}(x), \beta \times v_{A}(x)\right\rangle \mid x \in E\right\}, \\
& H_{\alpha, \beta}(A)=\left\{\left\langle x, \alpha \times \mu_{A}(x), v_{A}(x)+\beta \times \pi_{A}(x)\right\rangle \mid x \in E\right\}, \\
& H_{\alpha, \beta}^{*}(A)=\left\{\left\langle x, \alpha \times \mu_{A}(x), v_{A}(x)+\beta \times\left(1-\alpha \times \mu_{A}(x)-v_{A}(x)\right)\right\rangle \mid x \in E\right\}, \\
& J_{\alpha, \beta}(A)=\left\{\left\langle x, \mu_{A}(x)+\alpha \times \pi_{A}(x), \beta \times v_{A}(x)\right\rangle \mid x \in E\right\}, \\
& J_{\alpha, \beta}^{*}(A)=\left\{\left\langle x, \mu_{A}(x)+\alpha \times\left(1-\mu_{A}(x)-\beta \times v_{A}(x)\right), \beta \times v_{A}(x)\right\rangle \mid x \in E\right\},
\end{aligned}
$$

where $\alpha, \beta \in[0,1]$ are fixed numbers.

Obviously, operator $D_{\alpha}$ is an extension of the standard modal operators $\square$ and $\diamond$, because for each IFS A:

$$
\square A=D_{0}(A), \diamond A=D_{1}(A) .
$$

Operator $F_{\alpha, \beta}$ is an extension of the operator $D_{\alpha}$, because for each IFS $A$ :

$$
D_{\alpha}(A)=F_{\alpha, 1-\alpha}(A) .
$$

The rest of the operators do not have analogues in modal logic. Operators $D_{\alpha}, F_{\alpha, \beta}, \ldots$ are extensions of the operators $\square$ and $\diamond$, but they are objects of further extensions, described in [12].

It is important to mention that if $\alpha+\beta<1$, then for each fuzzy set $A$ and for each $x \in E$ :

$$
\begin{gathered}
\mu_{A}(x)+\alpha \times \pi_{A}(x)+v_{A}(x)+\beta \times \pi_{A}(x)=\mu_{A}(x)+v_{A}(x)+(\alpha+\beta) \times \pi_{A}(x) \\
<\mu_{A}(x)+v_{A}(x)+\pi_{A}(x)=1 .
\end{gathered}
$$

Therefore, $F_{\alpha \times \beta}(A)$ is not a fuzzy set. Hence, operator $F_{\alpha \times \beta}$ cannot be applied over $A$ without changing its status (from fuzzy set to IFS).

The situation with the other extended modal operators is similar.

The second group of modal operators can obtain T1FS-interpretation. They are defined sequentially, so each of the following operators is an extension of the previous ones. These operators are defined for each IFS $A$ by:

$$
\begin{gathered}
\boxplus A=\left\{\left\langle x, \frac{\mu_{A}(x)}{2}, \frac{v_{A}(x)+1}{2}\right\rangle \mid x \in E\right\}, \\
\rrbracket A=\left\{\left\langle x, \frac{\mu_{A}(x)+1}{2}, \frac{v_{A}(x)}{2}\right\rangle \mid x \in E\right\} ; \\
\bigoplus_{\alpha} A=\left\{\left\langle x, \alpha \times \mu_{A}(x), \alpha \times v_{A}(x)+1-\alpha\right\rangle \mid x \in E\right\}, \\
\bigotimes_{\alpha} A=\left\{\left\langle x, \alpha \times \mu_{A}(x)+1-\alpha, \alpha \times v_{A}(x)\right\rangle \mid x \in E\right\},
\end{gathered}
$$

where $\alpha \in[0,1]$;

$$
\begin{aligned}
& \bigoplus_{\alpha, \beta} A=\left\{\left\langle x, \alpha \times \mu_{A}(x), \alpha \times v_{A}(x)+\beta\right\rangle \mid x \in E\right\}, \\
& \bigotimes_{\alpha, \beta} A=\left\{\left\langle x, \alpha \times \mu_{A}(x)+\beta, \alpha \times v_{A}(x)\right\rangle \mid x \in E\right\},
\end{aligned}
$$

where $\alpha, \beta, \alpha+\beta \in[0,1]$.

$$
\bigoplus_{\alpha, \beta, \gamma} A=\left\{\left\langle x, \alpha \times \mu_{A}(x), \beta \times v_{A}(x)+\gamma\right\rangle \mid x \in E\right\},
$$




$$
\bigotimes_{\alpha, \beta, \gamma} A=\left\{\left\langle x, \alpha \times \mu_{A}(x)+\gamma, \beta \times v_{A}(x)\right\rangle \mid x \in E\right\},
$$

where $\alpha, \beta, \gamma \in[0,1]$ and $\max (\alpha, \beta)+\gamma \leq 1$;

$$
\bullet_{\alpha, \beta, \gamma, \delta} A=\left\{\left\langle x, \alpha \times \mu_{A}(x)+\gamma, \beta \times v_{A}(x)+\delta\right\rangle \mid x \in E\right\},
$$

where $\alpha, \beta, \gamma, \delta \in[0,1]$ and $\max (\alpha, \beta)+\gamma+\delta \leq 1$.

$$
\begin{gathered}
\oslash_{\alpha, \beta, \gamma, \delta, \varepsilon, \zeta} A=\left\{\left\langlex, \alpha \times \mu_{A}(x)-\varepsilon \times v_{A}(x)+\gamma,\right.\right. \\
\left.\left.\beta \times v_{A}(x)-\zeta \times \mu_{A}(x)+\delta\right\rangle \mid x \in E\right\},
\end{gathered}
$$

where $\alpha, \beta, \gamma, \delta, \varepsilon, \zeta \in[0,1]$ and

$$
\begin{aligned}
& \max (\alpha-\zeta, \beta-\varepsilon)+\gamma+\delta \leq 1, \\
& \min (\alpha-\zeta, \beta-\varepsilon)+\gamma+\delta \geq 0
\end{aligned}
$$

(see [12]).

In the next section, we discuss the modification of these operators for the T1FS-case.

The rest two groups of modal operators (see $[25,26]$ ) cannot be transformed for the T1FS-case, and will not be a subject of discussion here.

The basic intuitionistic fuzzy level operators (e.g., [12]) are:

$$
\begin{aligned}
& P_{\alpha, \beta}(A)=\left\{\left\langle x, \max \left(\alpha, \mu_{A}(x)\right), \min \left(\beta, v_{A}(x)\right)\right\rangle \mid x \in E\right\}, \\
& Q_{\alpha, \beta}(A)=\left\{\left\langle x, \min \left(\alpha, \mu_{A}(x)\right), \max \left(\beta, v_{A}(x)\right)\right\rangle \mid x \in E\right\},
\end{aligned}
$$

for $\alpha, \beta \in[0,1]$ and $\alpha+\beta \leq 1$.

The degrees of membership and non-membership of the elements of a given universe to its subset can be directly changed by these operators. These operators are a standard extension of the existing operators in T1FS theory.

Following [12], we introduce two operators that are analogous to the topological operators of closure and interior (e.g., [27,28]). They were defined in October 1983 by the author, and their basic properties were studied. Three years later, the relations between the modal and the topological operators over IFSs were studied (see [11]). These operators are defined for every IFS $A$ by:

$$
\begin{aligned}
& \mathcal{C}(A)=\left\{\left\langle x, \sup _{y \in E} \mu_{A}(y), \inf _{y \in E} v_{A}(y)\right\rangle \mid x \in E\right\}, \\
& \mathcal{I}(A)=\left\{\left\langle x, \inf _{y \in E} \mu_{A}(y), \sup _{y \in E} v_{A}(y)\right\rangle \mid x \in E\right\} .
\end{aligned}
$$

Since the beginning of the new century, these operators have been the objects of certain extensions. Six of these extensions are as follows (see [12]):

$$
\begin{gathered}
\mathcal{C}_{\mu}(A)=\left\{\left\langle x, \sup _{y \in E} \mu_{A}(y), \min \left(1-\sup _{y \in E} \mu_{A}(y), v_{A}(x)\right)\right\rangle \mid x \in E\right\}, \\
\mathcal{C}_{v}(A)=\left\{\left\langle x, \mu_{A}(x), \inf _{y \in E} v_{A}(y)\right\rangle \mid x \in E\right\}, \\
\mathcal{I}_{\mu}(A)=\left\{\left\langle x, \inf _{y \in E} \mu_{A}(y), v_{A}(x)\right\rangle \mid x \in E\right\}, \\
\mathcal{I}_{v}(A)=\left\{\left\langle x, \min \left(1-\sup _{y \in E} v_{A}(y), \mu_{A}(x)\right), \sup _{y \in E} v_{A}(y)\right\rangle \mid x \in E\right\}, \\
\mathcal{C}_{\mu}^{*}(A)=\left\{\left\langle x, \min \left(\sup _{y \in E} \mu_{A}(y), 1-v_{A}(x)\right), \min \left(1-\sup _{y \in E} \mu_{A}(y), v_{A}(x)\right)\right\rangle \mid x \in E\right\},
\end{gathered}
$$




$$
\mathcal{I}_{v}^{*}(A)=\left\{\left\langle x, \min \left(1-\sup _{y \in E} v_{A}(y), \mu_{A}(x)\right), \min \left(\sup _{y \in E} v_{A}(y), 1-\mu_{A}(x)\right)\right\rangle \mid x \in E\right\} .
$$

IFSs are extended in some directions (e.g., [11]): intuitionistic $L$-fuzzy sets, interval-valued IFSs, IFSs over different universes, temporal IFSs, multidimensional IFSs, IFSs of $n$-type. Now, 30 years after the introduction of this last extension, some authors have incorrectly re-branded them as "Pythagorean fuzzy sets", repeating the results from [29].

While the intuitionistic L-fuzzy sets are a direct extension of $L$-fuzzy sets [2] and the interval-valued IFSs are an extension of interval-values fuzzy sets [30], the rest extensions are the original ones (i.e., having no fuzzy sets analogues). Moreover, they cannot be transformed to T1FS-forms. For example, the IFSs of $n$-type have the form

$$
A=\left\{\left\langle x, \mu_{A}(x), v_{A}(x)\right\rangle \mid x \in E\right\},
$$

where

$$
0 \leq \mu_{A}(x)^{n}+v_{A}(x)^{n} \leq 1 .
$$

In the T1FS-case, the above inequality is transformed to $0 \leq \mu_{A}(x)^{n} \leq 1$, which is equivalent to $0 \leq \mu_{A}(x) \leq 1$ when $n>0$.

\section{What in IFS Theory Can Be Transformed to T1FS Theory?}

As we mentioned in Section 2, the first IFS-interpretation (Figures 1 and 2) is a direct extension of T1FS-interpretation, while the interpretations from Figure 3 can be transformed to the T1FS-case. The next interpretations from Figures $4-8$ cannot be used in the T1FS-case, while the latest interpretations (from Figure 9) can be used in the T1FS theory. It will have the form from Figure 11.

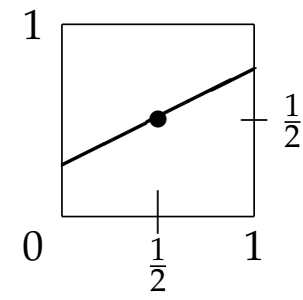

Figure 11. Geometrical interpretation of a T1FS element.

As we mentioned above, IFS-operations can be transformed to T1FS-operations, but many of them will coincide. For example, if we transform the two IFS-implications, defined for two IFSs $A$ and $B$ :

$$
\begin{gathered}
A \rightarrow_{3} B=\left\{\left\langlex, 1-\left(1-\mu_{B}(x)\right) \times \operatorname{sg}\left(\mu_{A}(x)-\mu_{B}(x)\right),\right.\right. \\
\left.\left.v_{B}(x) \times \operatorname{sg}\left(\mu_{A}(x)-\mu_{B}(x)\right)\right\rangle \mid x \in E\right\}
\end{gathered}
$$

and

$$
\begin{gathered}
A \rightarrow_{11} B=\left\{\left\langlex, 1-\left(1-\mu_{B}(x)\right) \times \operatorname{sg}\left(\mu_{A}(x)-\mu_{B}(x)\right),\right.\right. \\
\left.\left.v_{B}(x) \times \operatorname{sg}\left(\mu_{A}(x)-\mu_{B}(x)\right) \times \operatorname{sg}\left(v_{B}(x)-v_{A}(x)\right)\right\rangle \mid x \in E\right\}
\end{gathered}
$$

to T1FS-forms (i.e., when $A$ and $B$ are T1FSs), both sets $\left(A \rightarrow_{3} B\right.$ and $\left.A \rightarrow_{11} B\right)$ will coincide, obtaining the form

$$
A \rightarrow B=\left\{\left\langle x, 1-\left(1-\mu_{B}(x)\right) \times \operatorname{sg}\left(\mu_{A}(x)-\mu_{B}(x)\right)\right\rangle \mid x \in E\right\} .
$$

The same is the situation with the IFS-negations. For example, if we transform the three IFS-negations for the IFS $A$

$$
\neg_{1} A=\left\{\left\langle x, v_{A}(x), \mu_{A}(x)\right\rangle \mid x \in E\right\},
$$




$$
\begin{aligned}
\neg_{3} A= & \left\{\left\langle x, v_{A}(x), \mu_{A}(x) \times v_{A}(x)+\mu_{A}(x)^{2}\right\rangle \mid x \in E\right\}, \\
& \neg_{4} A=\left\{\left\langle x, v_{A}(x), 1-v_{A}(x)\right\rangle \mid x \in E\right\}
\end{aligned}
$$

to T1FS-forms (i.e., for a T1FS $A$ ), they will coincide, obtaining the form

$$
\neg A=\left\{\left\langle x, v_{A}(x)\right\rangle \mid x \in E\right\}=\left\{\left\langle x, 1-\mu_{A}(x)\right\rangle \mid x \in E\right\} .
$$

Obviously, the modal operators from the first group $\left(\square, \diamond, D_{\alpha}, \ldots\right)$ make no sense in the T1FS-case, while some of the operators from the second group can obtain T1FS-forms. Now, they will obtain the following forms:

$$
\begin{gathered}
\boxplus A=\left\{\left\langle x, \frac{\mu_{A}(x)}{2}\right\rangle \mid x \in E\right\}, \\
\bigotimes A=\left\{\left\langle x, \frac{\mu_{A}(x)+1}{2}\right\rangle \mid x \in E\right\} ; \\
\bigoplus_{\alpha} A=\left\{\left\langle x, \alpha \times \mu_{A}(x)\right\rangle \mid x \in E\right\}, \\
\bigotimes_{\alpha} A=\left\{\left\langle x, \alpha \times \mu_{A}(x)+1-\alpha\right\rangle \mid x \in E\right\},
\end{gathered}
$$

where $\alpha \in[0,1]$;

$$
\begin{gathered}
\bigoplus_{\alpha, \beta} A=\left\{\left\langle x, \alpha \times \mu_{A}(x)\right\rangle \mid x \in E\right\}, \\
\bigotimes_{\alpha, \beta} A=\left\{\left\langle x, \alpha \times \mu_{A}(x)+\beta\right\rangle \mid x \in E\right\},
\end{gathered}
$$

where $\alpha, \beta, \alpha+\beta \in[0,1]$.

$$
\begin{gathered}
\bigoplus_{\alpha, \beta, \gamma} A=\left\{\left\langle x, \alpha \times \mu_{A}(x)\right\rangle \mid x \in E\right\}, \\
\bigotimes_{\alpha, \beta, \gamma} A=\left\{\left\langle x, \alpha \times \mu_{A}(x)+\gamma\right\rangle \mid x \in E\right\},
\end{gathered}
$$

where $\alpha, \beta, \gamma \in[0,1]$ and $\max (\alpha, \beta)+\gamma \leq 1$;

$$
\bullet_{\alpha, \beta, \gamma, \delta} A=\left\{\left\langle x, \alpha \times \mu_{A}(x)+\gamma\right\rangle \mid x \in E\right\},
$$

where $\alpha, \beta, \gamma, \delta \in[0,1]$ and $\max (\alpha, \beta)+\gamma+\delta \leq 1$.

$$
\square_{\alpha, \beta, \gamma, \delta, \varepsilon, \zeta} A=\left\{\left\langle x, \alpha \times \mu_{A}(x)-\varepsilon \times v_{A}(x)+\gamma\right\rangle \mid x \in E\right\},
$$

where $\alpha, \beta, \gamma, \delta, \varepsilon, \zeta \in[0,1]$ and

$$
\begin{aligned}
& \max (\alpha-\zeta, \beta-\varepsilon)+\gamma+\delta \leq 1, \\
& \min (\alpha-\zeta, \beta-\varepsilon)+\gamma+\delta \geq 0 .
\end{aligned}
$$

We see immediately that

$$
\begin{gathered}
\bigoplus_{\alpha} A=\uplus_{\alpha, \beta} A=\bigoplus_{\alpha, \beta, \gamma} A, \\
\bigotimes_{\alpha, \beta, \gamma} A=\bullet_{\alpha, \beta, \gamma, \delta} A .
\end{gathered}
$$

On the other hand, some of the operator arguments must be omitted. So, in the T1FS-case, these operators will obtain, respectively, the forms:

$$
\begin{gathered}
\boxplus A=\left\{\left\langle x, \frac{\mu_{A}(x)}{2}\right\rangle \mid x \in E\right\}, \\
\bigotimes A=\left\{\left\langle x, \frac{\mu_{A}(x)+1}{2}\right\rangle \mid x \in E\right\} ; \\
\boxplus_{\alpha} A=\left\{\left\langle x, \alpha \times \mu_{A}(x)\right\rangle \mid x \in E\right\},
\end{gathered}
$$




$$
\bigotimes_{\alpha} A=\left\{\left\langle x, \alpha \times \mu_{A}(x)+1-\alpha\right\rangle \mid x \in E\right\},
$$

where $\alpha \in[0,1]$;

$$
\bigotimes_{\alpha, \beta} A=\left\{\left\langle x, \alpha \times \mu_{A}(x)+\beta\right\rangle \mid x \in E\right\},
$$

where $\alpha, \beta, \alpha+\beta \in[0,1]$.

$$
\bigcirc_{\alpha, \gamma, \varepsilon} A=\left\{\left\langle x, \alpha \times \mu_{A}(x)-\varepsilon \times v_{A}(x)+\gamma\right\rangle \mid x \in E\right\},
$$

where $\alpha, \gamma, \varepsilon, \in[0,1]$ and

$$
\begin{aligned}
& \alpha+\gamma \leq 1, \\
& \gamma-\varepsilon \geq 0 .
\end{aligned}
$$

The topological operators can also be reduced to the T1FS-case, as follows:

$$
\begin{gathered}
\mathcal{C}(A)=\left\{\left\langle x, \sup _{y \in E} \mu_{A}(y)\right\rangle \mid x \in E\right\}, \\
\mathcal{I}(A)=\left\{\left\langle x, \inf _{y \in E} \mu_{A}(y)\right\rangle \mid x \in E\right\}, \\
\mathcal{C}_{\mu}(A)=\left\{\left\langle x, \sup _{y \in E} \mu_{A}(y)\right\rangle \mid x \in E\right\}, \\
\mathcal{C}_{v}(A)=\left\{\left\langle x, \mu_{A}(x)\right\rangle \mid x \in E\right\}, \\
\mathcal{I}_{\mu}(A)=\left\{\left\langle x, \inf _{y \in E} \mu_{A}(y)\right\rangle \mid x \in E\right\}, \\
\mathcal{I}_{v}(A)=\left\{\left\langle x, \min \left(1-\sup _{y \in E} v_{A}(y), \mu_{A}(x)\right)\right\rangle \mid x \in E\right\}, \\
\mathcal{C}_{\mu}^{*}(A)=\left\{\left\langle x, \min \left(\sup _{y \in E} \mu_{A}(y), 1-v_{A}(x)\right)\right\rangle \mid x \in E\right\}, \\
\mathcal{I}_{v}^{*}(A)=\left\{\left\langle x, \min \left(1-\sup _{y \in E} v_{A}(y), \mu_{A}(x)\right)\right\rangle \mid x \in E\right\},
\end{gathered}
$$

where, again, $v_{A}(x)=1-\mu_{A}(x)$. Therefore,

$$
\begin{aligned}
& \mathcal{C}(A)=\mathcal{C}_{\mu}(A), \\
& \mathcal{I}(A)=\mathcal{I}_{\mu}(A),
\end{aligned}
$$

$\mathcal{C}_{v}$ is deprecated. The same is the situation with the last three topological operators, because:

$$
\begin{gathered}
\mathcal{I}_{v}(A)=\left\{\left\langle x, \min \left(1-\sup _{y \in E}\left(1-\mu_{A}(y)\right), \mu_{A}(x)\right)\right\rangle \mid x \in E\right\} \\
=\left\{\left\langle x, \min \left(1-\left(1-\inf _{y \in E}\left(\mu_{A}(y)\right)\right), \mu_{A}(x)\right)\right\rangle \mid x \in E\right\} \\
=\left\{\left\langle x, \min _{y \in E}\left(\inf _{y \in E}\left(\mu_{A}(y)\right), \mu_{A}(x)\right)\right\rangle \mid x \in E\right\} \\
=\left\{\left\langle x, \inf _{y \in E} \mu_{A}(x)\right\rangle \mid x \in E\right\}=\mathcal{I}(A), \\
\mathcal{C}_{\mu}^{*}(A)=\left\{\left\langle x, \min \left(\sup _{y \in E} \mu_{A}(y), \mu_{A}(x)\right)\right\rangle \mid x \in E\right\} \\
=\left\{\left\langle x, \mu_{A}(x)\right\rangle \mid x \in E\right\}=A, \\
\mathcal{I}_{v}^{*}(A)=\left\{\left\langle x, \min \left(1-\sup _{y \in E}\left(1-\mu_{A}(y)\right), \mu_{A}(x)\right)\right\rangle \mid x \in E\right\}
\end{gathered}
$$




$$
\begin{gathered}
=\left\{\left\langle x, \min \left(1-\left(1-\inf _{y \in E} \mu_{A}(y)\right), \mu_{A}(x)\right)\right\rangle \mid x \in E\right\} \\
=\left\{\left\langle x, \min \left(\inf _{y \in E} \mu_{A}(y), \mu_{A}(x)\right)\right\rangle \mid x \in E\right\} \\
=\left\{\left\langle x, \inf _{y \in E} \mu_{A}(y)\right\rangle \mid x \in E\right\}=\mathcal{I}(A) .
\end{gathered}
$$

Therefore, only the first two topological operators $(\mathcal{C}$ and $\mathcal{I})$ make sense in the T1FS-case.

\section{Conclusions}

IFSs is an extension of T1FS. The theory containing new operations, relations, and operators that essentially extend the operators defined over T1FSs. In this paper, we discussed which of them can be and which cannot be transformed over T1FSs. So, now it is clear that there are possibilities for development in new directions of the T1FSs.

Acknowledgments: The author is grateful to the anonymous reviewers for their very valuable comments. The present research has been supported by the Bulgarian National Science Fund under Grant Ref. No. DFNI-I-02-5 "InterCriteria Analysis: A New Approach to Decision Making".

Conflicts of Interest: The authors declare no conflict of interest.

\section{References}

1. Zadeh, L. Fuzzy sets. Inf. Control 1965, 8, 338-353.

2. Goguen, J. L-fuzzy sets. J. Math. Anal. Appl. 1967, 18, 145-174.

3. Zadeh, L. The concept of a linguistic variable and its application to approximate reasoning. Inf. Sci. 1975, 8, 199-249.

4. Pawlak, Z. Rough Sets Research Report PAS 431; Institute of Computer Science, Polish Academy of Sciences: Warsaw, Poland, 1981.

5. Pawlak, Z. Rough sets. Int. J. Comput. Inf. Sci. 1982, 11, 341-356.

6. Atanassov, K. Intuitionistic fuzzy sets. In Proceedings of the VII ITKR's Session, Sofia, Bulgaria, 7-9 June 1983 (Deposed in Central Sci.- Techn. Library of Bulg. Acad. of Sci., 1697/84) (in Bulgaria); reprinted in Int. J. Bioautom. 2016, 20, S1-S6.

7. Castillo, O.; Melin, P. Type-2 Fuzzy Logic: Theory and Applications; Springer: Berlin, Germany, 2008.

8. Gonzalez, C.I.; Melin, P.; Castro, J.R.; Castillo, O. Edge Detection Methods Based on Generalized Type-2 Fuzzy Logic; Springer: Cham, Switzerland, 2017.

9. Ponce-Cruz, P.; Molina, A.; MacCleery, B. Fuzzy Logic Type-1 and Type-2 Based on LabVIEW FPGA; Springer: Cham, Switzerland, 2016.

10. Kaufmann, A. Introduction a La Theorie Des Sour-Ensembles Flous; Masson: Paris, France, 1977.

11. Atanassov, K. Intuitionistic Fuzzy Sets; Springer: Heidelberg, Germany, 1999.

12. Atanassov, K. On Intuitionistic Fuzzy Sets Theory; Springer: Berlin, Germany, 2012.

13. Danchev, S. A new geometrical interpretation of some concepts in the intuitionistic fuzzy logics. Notes Intuit. Fuzzy Sets 1995, 1, 116-118.

14. Szmidt, E. Applications of Intuitionistic Fuzzy Sets in Decision Making. Ph.D. Thesis, Technical University, Sofia, Bulgaria, 2000.

15. Szmidt, E.; Kacprzyk, J. Similarity of intuitionistic fuzzy sets and the Jaccard coefficient. In Proceedings of the Tenth International Conference IPMU'2004, Perugia, Italy, 4-9 July 2004; Volume 2, pp. 1405-1412.

16. Szmidt, E.; Kacprzyk, J. New measures of entropy for intuitionistic fuzzy sets. Notes Intuit. Fuzzy Sets 2005, $11,12-20$.

17. Atanassov, K. New geometrical interpretation of the intuitionistic fuzzy pairs. Notes Intuit. Fuzzy Sets 2016, $22,12-18$.

18. Atanassov, K. Intuitionistic Fuzzy Logics; Springer: Cham, Switzerland, 2017.

19. Atanassov, K.; Szmidt, E.; Kacprzyk, J. New Fodor's type of intuitionistic fuzzy implication and negation. Notes Intuit. Fuzzy Sets 2016, 22, 1-8. 
20. Atanassov, K.; Szmidt, E.; Kacprzyk, J. Intuitionistic fuzzy implication $\rightarrow$ 187. Notes Intuit. Fuzzy Sets 2017, $23,37-43$.

21. Atanassov, K.; Szmidt, E.; Kacprzyk, J. Intuitionistic fuzzy implication $\rightarrow 188$. Notes Intuit. Fuzzy Sets 2017, 23, 6-13.

22. Atanassova, L. Intuitionistic fuzzy implication $\rightarrow$ 189. Notes Intuit. Fuzzy Sets 2017, 23, 14-20.

23. Brouwer, L.E.J. Collected Works; North Holland: Amsterdam, The Netherlands, 1975; Volume 1.

24. Van Dalen, D. (Ed.) Brouwer's Cambridge Lectures on Intuitionism; Cambridge University Press: Cambridge, UK, 1981.

25. Atanassov K.; Gargov, G. Intuitionistic fuzzy logic operators of a set theoretical type. In Proceedings of the First Workshop on Fuzzy Based Expert Systems, Sofia, Bulgaria, 28-30 September 1994; pp. 39-42.

26. Atanassova, V.; Doukovska, L. Compass-and-straightedge constructions in the intuitionistic fuzzy interpretational triangle: Two new intuitionistic fuzzy modal operators. Notes Intuit. Fuzzy Sets 2017, 23, 1-7.

27. Kuratowski, K. Topology; Academies Press: New York, NY, USA, 1966; Volume 1.

28. Yosida, K. Functional Analysis; Springer-Verlag: Berlin, Germany, 1965.

29. Atanassov, K. Geometrical interpretation of the elements of the intuitionistic fuzzy objects. Preprint IM-MFAIS-1-89, Sofia, Bulgaria, 1989; reprinted in Int. J. Bioautom. 2016, 20, S27-S42.

30. Gorzalczany, M. A method of inference in approximate reasoning based on interval-valued fuzzy sets. Fuzzy Sets Syst. 1987, 21, 1-17.

(C) 2017 by the author. Licensee MDPI, Basel, Switzerland. This article is an open access article distributed under the terms and conditions of the Creative Commons Attribution (CC BY) license (http:/ / creativecommons.org/licenses/by/4.0/). 